\title{
Sanfte Beckenbodenarbeit im Frühwochenbett
}

\author{
Osariere-Claudia Röthlisberger, MSc Midwifery, Richterswil bei Zürich
}

\section{Eine explorative qualitative Untersuchung erforscht die Sicht der Nutzerin auf das Hebammenangebot einer sanften Beckenbodenarbeit im Frühwochenbett. Die Ergeb- nisse motivieren dazu, die Frauen individuell anzuleiten.}

Die Gesundheit von Frauen ist oft durch Beckenbodenbeschwerden beeinträchtigt. Beckenbodendysfunktionen treten häufig erstmals im Leben einer Frau in Folge von Schwangerschaft und Geburt auf [1,2]. Je nach Quelle variiert die Prävalenz; in einer australischen Kohortenstudie sind von 1507 Erstparae $47 \%$ von Harninkontinenz und 18\% von Stuhlinkontinenz innerhalb der ersten $12 \mathrm{Mo-}$ nate postpartum betroffen [16].

Das Beckenbodengewebe wird durch die peripartalen Veränderungen stark beansprucht und noch in der Exogestation (die ersten 9 Monate nach der Geburt) ist es geschwächt und besonders anfällig für Überbelastung. Zudem ist das Perineum nach der Geburt noch lange empfindlich bis schmerzend [2]. Die Früherkennung von Wöchnerinnen mit Symptomen von Inkontinenz oder anderen Beckenbodendysfunktionen bzw. mit Risiken dafür und das Einleiten entsprechender Maßnahmen ist essentiell $[4,17]$.

Die Reorganisation der Muskelfasern wird unterstützt durch Entlastung des Beckenbodengewebes, durch achtsame Übungen zur Wahrnehmung und sanfte Bewegungsreize beginnend in den ersten 10 Tagen pp [4]. Dies gilt auch bei unauffälligen Verläufen und unabhängig vom Geburtsmodus [5], denn wenn physiologische Reize auf das Gewebe einwirken können, wird die Regeneration begünstigt durch die Organisation und Ausrichtung der Kollagenmoleküle des Gewebes in die Funktionsrichtung. So

kann normales Gewebe mit weniger Narbengewebe entstehen. Dies betrifft die sichtbaren Geburtsverletzungen sowie die unsichtbaren Beckenbodenstrukturen [6]. Tab. 1 zeigt die Physiologie der Wundheilung.

Anleitung und Information, wie junge Mütter im Alltag ihren Beckenboden schützen und stärken, d.h. nach Schwangerschaft und Geburt bzw. Kaiserschnitt aufbauen können, gehören zu den professionellen Aufgaben der Hebamme im

Wochenbett. Dennoch erhält diese wichtige frühe Beckenbodenarbeit (BBA) oft nicht gebührend Raum innerhalb der Begleitung $[7,8]$. Den Frauen selber ist das Wohlergehen ihres Kindes meist wichtiger, als ihre eigene Befindlichkeit und Gesundheit [16].

Aufgrund der Physiologie und emotionaler Aspekte stehen im Frühwochenbett die Entlastung des Beckenbodengewebes, achtsame Übungen zur Beckenbodenwahrnehmung und sanfte

Tab. 1 Phasen der Wundheilung [4, 6].

\section{Phase}

Entzündungsphase

0.-5.Tag

Maßnahmen

Proliferationsphase

5.-21. Tag

Maßnahmen

Konsolidierungsphase 21.-60. Tag Maßnahmen

Organisations- und Umbauphase 60.-360.Tag
Die Entzündungsphase wird unterteilt in vaskuläre Phase und zelluläre Phase.

Die mechanische Belastbarkeit ist reduziert. Möglichst Entlastung des Beckenbodens, häufiges Liegen. Durchblutungsförderung, Atemlenkung und Spür-Übungen.

Fließende, ineinander übergehende Reparatur- und Reorganisationsprozesse.

Dosierte Bewegungen stimulieren die Bildung eines gut organisierten Gewebes.

Ab dem ca.10.-21. Tag, idealer Zeitraum für dosierte Wochenbett-Rückbildungsgymnastik und aufbauende Beckenbodenarbeit (nach Kaiserschnitt ab dem 14. Tag).

Weiterhin hohe Kollagensynthese. Kollagenumbau zu belastungsfähigerem und flexiblerem Gewebe.

Aufnahme von angepassten sportlichen Aktivitäten gemäß dem Befinden, respektieren von Schmerzgrenzen und Ermüdungszeichen nach Aufbau der Beckenbodenmuskulatur.

hohe Kollagensynthese, ab 150.-360. Tag abnehmende Fibroblaste, zunehmende Belastbarkeit und Reißfestigkeit des Gewebes. 
Bewegungsreize, um die Wundheilungsprozesse zu unterstützen, im Vordergrund $[4,9]$.

\section{Sanfte Beckenbodenarbeit}

Die physiologischen Heilungsprozesse im Beckenbodengewebe und der Geburtsverletzungen zu unterstützen bedeutet, diesen Bereich zu entlasten, aber auch frühzeitig angepasste Bewegungsreize anzubieten, langsam steigernd gemäß den Gesetzen der Wundheilung; im frühen Wochenbett ist noch kein starkes Beckenbodentraining angezeigt und vor allem ist die Aktivierung der geraden Bauchmuskeln in Rückenlage zu vermeiden.

Die beiden Aspekte Schützen und Stärken begünstigen die physiologische Regeneration des Beckenbodens [4,5]. Daher sollen alle Wöchnerinnen Anleitung und Information zur sanften BBA erhalten, unabhängig von manifesten Symptomen einer Beckenbodendysfunktion oder asymptomatischer Genesung.

\section{Frühe sanfte Beckenbodenarbeit}

- ist eine achtsame therapeutische und präventive Intervention;

- umfasst Anleitung und Information zum Schutz und Stärkung des Beckenbodens:

- Schonung und Entlastung des Beckenausgangs zur Vermeidung von unnötigem Druck auf Beckenbodengewebe und Perineum

- Optimierung des Bewegungsverhalten und der Körperhaltung

- Beckenbodenwahrnehmung und Atemlenkung mit Einsatz von Phonemen (Lautelemente) [4]

- sanfte Übungen für den Beckenboden mit co-aktiven Bauchmuskeln $[10,11]$ in Entlastungsposition

\section{Forschungsvorgehen}

Die vorliegende Untersuchung befasste sich mit folgenden Fragen:

- Welche Bedeutung hat die Becken-

Tab. 2 Ein- und Ausschlusskriterien der Untersuchung.

\section{Einschlusskriterien}

2-8 Wochen pp

Jeder Geburtsmodus

Erst- und Mehrpara

Deutsch sprechende Wöchnerinnen

Betreuung durch die Hebamme im Wochenbett mit Thema Beckenboden, gemäß dem üblichen Angebot der Hebamme

bodenarbeit innerhalb der Hebammenbetreuung im Frühwochenbett für Wöchnerinnen?

- Was hilft Wöchnerinnen, die Beckenbodenarbeit in ihren Familienalltag zu integrieren und außerhalb der Hebammenbetreuung fortzuführen?

Ziel war es, Anregungen für eine salutogene Betreuung zu gewinnen.

In der qualitativen Untersuchung wurde die frühe Beckenbodenarbeit aus der Perspektive der Nutzerin untersucht. Die Datenerhebung erfolgte im Frühsommer 2013 mittels problemzentrierten Leitfadeninterviews bei den Wöchnerinnen zu Hause im Großraum Zürich ( $n=6$, Interviewdauer 30-60 Min.). Tab. 2 zeigt die Ein- und Ausschlusskriterien der Untersuchung. Ethische Aspekte und der Datenschutz wurden während des ganzen Forschungsprozesses respektiert [3].

Die 6 Teilnehmerinnen in dem Convenience-Sampling (nicht-repräsentative Auswahl) haben einen homogenen soziodemografischen Hintergrund (30 bis 37 Jahre alt, alle verheiratet und akademische Bildung). Für die eine Hälfte der Frauen war es das 1 . Kind, für die andere das zweite. Die Teilnehmerinnen waren teils von prä- und/oder postpartaler Inkontinenz betroffen. Sie hatten eine Spontangeburt bzw. eine vaginal-operative Geburt, keine Teilnehmerin hatte einen Kaiserschnitt oder erhebliche gesundheitliche Probleme. Akquiriert

\section{Ausschlusskriterien}

Am Geburtsort gezielte Einzel-Physiotherapie erhalten

Fremdsprachige Frauen, die sich nicht gut auf Deutsch mitteilen können

Bereits Teilnahme an einem Rückbildungskurs

wurden die Studienteilnehmerinnen von Hebammenkolleginnen, die in der Betreuung immer Wochenbettgymnastik und die Thematik Beckenboden schützen und stärken einschließen.

Die Datenanalyse der transkribierten Audioaufnahmen wurde gemäß der inhaltlich strukturierenden qualitativen Inhaltsanalyse nach Mayring durchgeführt. Die Entwicklung des Kategoriensystems erfolgte deduktiv und induktiv in einem zirkulären Prozess. Beispielsweise wurden Dimensionen des SF-36 [2] (Fragebogen zur gesundheitsbezogenen Lebensqualität) und Aspekte aus dem saluto-physiologischen Betreuungsmodell [12] adaptiert. Alle Kategorien wurden in 6 Themen zusammengefasst.

\section{Ausgewählte Ergebnisse}

\section{Bedürfnis nach „Anleitung“}

Die frühe sanfte Beckenbodenarbeit entspricht einem Bedürfnis der Wöchnerinnen, auch wenn sie es während der Betreuung nicht an 1. Stelle als Anliegen an die Hebamme formulierten, da die Sorge um das Neugeborene und teils Stillschwierigkeiten im Vordergrund standen.

Alle Interviewpartnerinnen erachten das Angebot als sehr wichtigen Anteil der Hebammenarbeit während der Wochenbettbetreuung. Bei den befragten Frauen, 
die das 2. Kind geboren haben, stehen dabei Beschwerden und Inkontinenz im Vordergrund sowie der Wunsch, die körperliche Funktionsfähigkeit wiederzuerlangen, um ihre „Mutterrolle“ wahrnehmen und den Arbeitsalltag mit Kindern bewältigen zu können.

Die befragten Mütter, die erstmalig geboren haben, äußern ihren Bedarf nach Anleitung und Information vor allem, um sicher zu sein, dass sie Haltung, Bewegungsablauf oder Übungen richtig ausführen. Sie wollen mit einer Fachperson über die körperlichen Veränderungen sprechen und sich über das passende Maß an körperlicher Aktivität vergewissern.

„....wenn dann noch jemand vorbei kommt und das zeigt, wie das wirklich gehen sollte; ...weil, das habe ich nämlich genau immer falsch gemacht..."W3

„Bei der Hebamme kann man halt einfach auch nachfragen, wenn man sich nicht sicher ist, ob man es richtig macht, ob man es richtig spürt oder eben auch nicht spürt. [...] was sie mir auch gesagt hat, ist das mit dem auf die Toilette gehen, dass man sich eben auch da Zeit lassen und nicht schnell, schnell pressen soll, sondern sich Zeit lassen, dass sich die Muskulatur selber entspannt. Und dann sich einfach die Zeit zu nehmen und einfach mal die Tür zuzulassen und warten, bis es kommt ... ich wusste das gar nicht, dass das so stark zusammenhängt und einen Einfluss hat, das war für mich ein neues Thema." W1

„Also im Spital wurde mir dieses Mal gar nichts gesagt, das fand ich ganz komisch. Also letztes Mal kam die Physiotherapeutin, und die hat einem ein paar Sachen gesagt. [...] gerade beim zweiten Kind braucht man es eigentlich fast mehr als beim Ersten, fand ich. Ja, weil man hat körperlich eben ganz andere Sachen, die man machen muss. Also nach dem alles geweitet wurde, und man muss dann wieder tragen, und das ist dann alles eigentlich noch nicht so stabil. [...] es hieß dann einfach [beim Austrittsgespräch], ich solle ihn anspannen. Aber ich stellte dann fest, das geht gar nicht. Also [die Hebamme] hatte mir dann eben gesagt, wie ich dann wieder anfangen kann." W5

Die frühe sanfte Beckenbodenarbeit wird von Teilnehmerinnen als besonders geeignet für die Wochenbettzeit beschrieben, da während dieser Zeit die körperliche Sensibilität erhöht ist.

\section{Gesundheit und zum vertrauten Selbst zurückfinden}

„...dass man das Gefühl hat, es geht wieder einmal um einen selber. Man setzt sich vielleicht wieder ein bisschen intensiver mit den eigenen Empfindungen und Bedürfnissen auseinander, was man sonst nicht macht, weil einen niemand danach fragt. Weil man halt einfach funktioniert für das Kind und was sonst einfach sonst zu machen ist.“W1

„...prinzipiell war es für mich, für meinen Körper, was nicht mit [meinem Sohn] zu tun hat, ...dass alles zusammenkommt, dass ich als Ganzes betrachtet wurde." W4

Die Interventionen werden als Gesundheitsförderung wahrgenommen und helfen zum vertrauten Selbst zurückzufinden. Sie geben den Interviewpartnerinnen das Gefühl, als Person wichtig zu sein und nicht nur als Mutter. Zudem verbinden die interviewten Frauen mit der Beckenbodenarbeit Prävention vor Inkontinenz und vor Komplikationen in einer nächsten Schwangerschaft.

Die Frauen äußern, dass ihnen die frühe sanfte Beckenbodenarbeit Gelegenheit 
gibt für die körperliche Regenerierung, die im Familienalltag einer jungen Mutter eher selten ist. Außerdem äußern sie, durch die BBA die Effektivität des eigenen präventiven Handelns wahrzunehmen und zu optimieren. Durch verbesserte Körperhaltung und Bewegungsabläufe werden z.B. Rückenschmerzen verhindert und das Wohlbefinden gesteigert.

Die zurückgewonnene körperliche Funktionsfähigkeit wirkt sich positiv aus auf Kontinenz und auf leichte körperliche Aktivitäten und erleichtert v.a. den Mehrparae die Alltagsbewältigung (z.B. Kinder tragen, trösten, wickeln, für Ausgleich zwischen den Geschwistern sorgen).

Die Intervention wirkt auf die emotionale Balance in der Wochenbettzeit und unterstützt Erstparae in Bezug auf ihr unbekanntes, verändertes Körpergefühl.

\section{Soziale Anpassung, Verhalten, Wochenbettkultur}

In den Interviewgesprächen wurden Auswirkungen der Beckenbodenarbeit auf das eigene Verhalten und auf das soziale Umfeld thematisiert. Bestärkt darin, sich als Wöchnerin vor Überlastungen zu schützen, delegierten Interviewpartnerinnen vermehrt Aufgaben. So entstehen Erholungsinseln im Familienalltag sowohl während als auch außerhalb der Hebammenbesuche.

„...wenn die Hebamme da ist und mit mir Beckenbodenübungen macht oder auch einfach nur fragt, das ist eine Auszeit von der Familie... Es ist emotional eine Erho- lungsphase, man hat ganz kurz Urlaub so diese halbe Stunde, Stunde, je nachdem, ob man sich jetzt nur um den Kleinen kümmert oder nur um mich oder um beide. "W6

\section{Instrumente haben}

Mit der sanften Beckenbodenarbeit während der frühen Wochenbettbetreuung erinnern oder erwerben sich die Wöchnerinnen kognitive wie auch kinästhetische Skills, die über die Betreuungszeit hinaus für sie verfügbar bleiben. Die verinnerlichten körperlichen Erfahrungen sind Instrumente, die sie auch später nutzen wollen.

„Man kann die Übungen ja auch während der [nächsten] Schwangerschaft machen.“ W6

Interviewpartnerinnen können sich besser an ihre Situation in der Postpartalzeit anpassen, ihren Beckenboden schonen und die Ansprüche an sich selbst zurücknehmen, z.B. sich nicht einem übersteigerten oder sogar falschen Trainingsprogramm zu unterziehen.

„...weil das war mir nicht bewusst, dass das schon etwas ist, dass das schon reicht am Anfang." W3

\section{Die Hebamme - Orientierung und Ansporn}

„...unter Anleitung eben, weil sonst würde man es ja nicht machen.“W4

„Weil, wenn man nichts gesagt kriegt, selbst wenn man es auf dem Papier hat... man macht es nicht."W6

„...weil man selbst kommt nicht dazu [...] weil der Alltag einen so schnell hat und einen die neue Situation auch überfordert [...]. Und wenn dann einer kommt und sagt, jetzt machen wir mal was, dann rafft man sich auf und dann merkt man auch, das geht ja vielleicht schon wieder oder ach ich hätte das jetzt probiert, das 
soll ich noch gar nicht, gut zu wissen." W6

Die Hebamme wird als wichtige äußere Ressource wahrgenommen. Sie ist nicht nur Vertrauensperson und im richtigen Moment ansprechbare Informationsträgerin, sondern Orientierungspunkt für die Wöchnerinnen. Bei ihr kann sie sich vergewissern, das beruhigt und bestärkt. Zudem ist die Hebamme Motivatorin und schafft während den Besuchen Zeit und Raum für angepasste Bewegung und Beckenbodenarbeit.

\section{Die Integration in den Familienalltag}

„...es waren leichte Übungen und Dinge, die man in den Alltag integrieren kann. Das war für mich wichtig, weil ich nicht eine Stunde Zeit habe, mich auszuklinken."W1

Beschwerden oder Angst vor erneuter Inkontinenz oder vor einer späteren Senkung sind Motivatoren für die Fortsetzung des beckenbodenschonenden Verhaltens und der Übungen, genügen aber meist alleine nicht. Hierfür muss jemand das Thema ansprechen, ihm Wichtigkeit beimessen und konkrete Vorschläge machen. Aus den Interviews geht hervor, dass einfache Übungen, die mit Alltagstätigkeiten verbunden werden können, eher weiterhin gemacht werden im Vergleich zu Übungssequenzen, die vom Alltag losgelöst sind. Außerdem sind Wiederholung und Erinnerungsstützen nötig und erwünscht, um die Nachhaltigkeit der Interventionen zu optimieren.

\section{Diskussion}

Mit dem kleinen Sampling konnte keine Sättigung bei den Themen erzielt werden. Es ist daher anzunehmen, dass nicht alle Themen identifiziert wurden. Limitierend ist außerdem, dass durch die Einschränkung auf die deutsche Sprache Informationen z.B. von Migrantinnen fehlen.
Zwei Erkenntnisse sind hervorzuheben: Die Einschätzung der befragten Wöchnerinnen, dass diese Interventionen der Hebamme für die Wochenbettzeit besonders geeignet ist aufgrund der erhöhten körperlichen Sensibilität und dass die frühe sanfte Beckenbodenarbeit einen Beitrag zur Wochenbettkultur leistet, indem sie die Lying-in-time unterstützt.

Das Bedürfnis der befragten Wöchnerinnen nach Absicherung, v.a. hinsichtlich korrekter Ausführung, bestätigen andere Untersuchungen [13]. Fehlendes Feedback und Informationsdefizite können zur Folge haben, dass die BBA nicht fortgeführt wird [14]. Um die Nachhaltigkeit zu verbessern, schlagen Bick et al. vor, gemeinsam mit der Wöchnerin Informationsmaterial zu lesen und anzuschauen und hierbei auf ihr Vorwissen Bezug zu nehmen [15].

Die Aussagen der Interviewpartnerinnen decken sich mit den Studienergebnissen von Chiarelli et al., dass eine gute Anleitung mit umfassender Information zu Wirkungsweise und Ziel von BBT und Instruktion zur korrekten Ausführung mit Überprüfung so wie Ermutigung zur regelmäßigen Durchführung notwendig sind [13].

Assessments zur Detektion von prädikativen Risikofaktoren resp. Symptomen für Beckenbodendysfunktionen sind bei jeder Wöchnerin vorzunehmen [17]. Risikofaktoren können Inkontinenz in der Schwangerschaft, hoher BMI und chronischer Husten, Nikotinabusus, prolongierte Geburt, kindliches Geburtsgewicht $>4000$ g, DR II-IV oder vaginal operative Entbindung sein. Vorübergehende Empfindungseinschränkungen im Beckenausgang in den ersten Tagen sowie eine herabgesetzte Reaktionsfähigkeit der Muskulatur kann durch eine Überdehnung der Nervenstruktur (N. pudendus) entstanden sein.

Bei ausgeprägter oder anhaltender Beckenbodendysfunktion $>2$ Wochen ist es wichtig, eine Physiotherapie zu veranlas- sen $[4,15]$. Um Motivation und Übungsgelegenheit in der Gruppe zu bekommen, ist die Empfehlung zu weiterführenden Rückbildungskursen und fortführendem Beckenbodentraining wichtig [9].

Frühe Information und Anleitung zu Schutz und Stärkung des Beckenbodens sind direkte Gesundheitsförderung über die Körperarbeit und die mental-kognitive Ebene, daher sind sie ein wichtiges ressourcenorientiertes Instrument in einer salutogenen Begleitung. Auch wenn für die Effektivität dieser Interventionen insbesondere bei asymptomatischen Frauen (noch) keine Daten verfügbar sind, sind es die Physiologie der Wundheilung und die Bedeutung für die Zielgruppe, die für die frühe Beckenbodenarbeit sprechen.

Seit Juli 2015 werden nun in der Schweiz Besuche nicht nur bis zum 10. Tag, sondern ähnlich wie in Deutschland, 10 bzw. 16 Termine bis 8 Wochen nach der Geburt von der Grundversicherung ohne ärztliche Verordnung vergütet.

Da Hebammen Frauen während einer Zeit begleiten, in der der Beckenboden sehr vulnerabel ist, liegt hier ein Präventionspotenzial für die maternale Gesundheit.

\section{Fazit für die Praxis}

- Individuell angepasste Beckenbodenarbeit in der Wochenbettbetreuung ist wichtig und nötig für alle Wöchnerinnen, auch für Frauen ohne Symptome oder Risikofaktoren.

- Eine sanfte Beckenbodenarbeit mit Schonung und Entlastung des Beckenausgangs beginnend im Frühwochenbett nutzt das Präventionspotenzial im bestgeeigneten Zeitraum (vulnerabler Beckenboden in der Regenerationsphase).

- Anleitung und Information, wie die Wöchnerin ihren Beckenboden schützen, aktivieren und entspannen kann, mit Überprüfung und Rückmeldung zur korrekten Ausführung 
geben Sicherheit, sind direkt umsetzbar und werden von den Frauen sehr geschätzt.

- Die Eins-zu-Eins-Betreuung in der häuslichen Wochenbettbetreuung ist ideal für die Information und Anleitung zur Beckenbodenarbeit.

- Die Wöchnerinnen orientieren sich stark an ihrer Hebamme, daraus ergibt sich eine hohe Verantwortung.

- Nachhaltigkeit wird gefördert durch gemeinsames Lesen und Erläutern von Informationsmaterial (Bild und Text).

- Wenn das Thema von der Hebamme als Fachperson wiederholt nachdrücklich eingebracht wird, mit Erinnerungsstützen und motivierenden Informationen, hat dies einen wesentlichen Einfluss auf das Fortführen.

- Wiederholte Information und Anleitung mit alltagstauglichen Übungen und Verhaltensanregungen, idealerweise angeknüpft an bereits Bekanntes, begünstigen die Integration in den Familienalltag.

Dieser Artikel basiert auf einer Masterarbeit im Studiengang „Angewandte Physiologie für Hebammen MSc Midwifery an der Fachhochschule Salzburg/ Puch; eingereicht von Osariere-Claudia Röthlisberger im September 2013.

\section{Literatur}

1 MacLennan AH et al. The prevalence of pelvic floor disorders and their relationship to gender, age, parity and mode of delivery. BJOG: An International Journal of Obstetrics and Gynaecology 2000; 107 (12); 1460-1470

2 Schäfers R. Subjektive Gesundheitseinschätzung gesunder Frauen nach der Geburt eines Kindes. Münster: MV-Verlag, 2011
3 Röthlisberger O-C. Sanfte und indirekte Beckenbodenarbeit im Frühwochenbett. Bedeutung dieser Hebammenarbeit aus der Sicht von jungen Müttern in Zürich-Agglomeration. Masterarbeit: Fachhochschule Salzburg/Puch, 2013

4 Tanzberger R et al. Der Beckenboden Funktion, Anpassung und Therapie. Das Tanzberger-Konzept. [Hrsg.] Renate Tanzberger und Gitta Wilke-Kaltenbach. München: Elsevier, Urban \& Fischer, 2009

5 Dumoulin C, Hay-Smith J. Pelvic floor muscle training versus no treatment, or inactive control treatments, for urinary incontinence in women. Cochrane Database. [Online] 20. Jan 2010

6 Van den Berg F. Angewandte Physiologie. Stuttgart: Thieme, 2003. Bd. 1

7 Schweizerischer Hebammenverband SHV. hebamme.ch. Qualitätsmanagement frei praktizierender Hebammen (fpH) Kundinnenbefragung 2009 [Online] 2010. [Zitat vom: 15. 02 2013.] http://www.hebamme.ch/x_dnld/quali/ Kundinnenbefragung_2009_d.pdf

8 hebamme.ch. Statistikbericht 2011. Statistik frei praktizierender Hebammen der Schweiz. Auswertung der Daten 2011. [Online] 2012. [Zitat vom: 16. 06 2013.] http://www.hebamme.ch/x_dnld/ stat/Statistikbericht_2011_d.pdf

9 Tacke L, Stüwe M. Wochenbett- und Rückbildungsgymnastik. 3. Auflage. Stuttgart: Hippokrates, 2013

10 Sapsford RR, Hodges PW et al. Co-activation of the abdominal and pelvic floor muscles during voluntary exercises. Neurourol Urodyn 2001; 20 (1): 31-42

11 Stauffer M, Zavagni-Roelly K. Vom Wochenbettturnen zur gezielten Intervention bei Risikowöchnerinnen. Physioactiv 2011; 3: 27-33

12 Schmid V. Schwangerschaft, Geburt und Mutterwerden. Ein Salutogenetisches Betreuungsmodell. Hannover: Erwin Staude, 2011
13 Chiarelli P, Murphy B, Cockburn J. Women's knowledge, practises, and intentions regarding correct pelvic floor exercises. Neurourol Urodyn 2003; 22 (3): 246-249

14 Gillard S, Shamley D. Factors motivationg women to commence and adhere to pelvic floor muscle exercises following a perineal tear at delivery: the influence of experience. Journal of the Association of Chartered Physiotherapists in Women's Health 2010; 106: 5-18

15 Bick D et al. Postnatal Care. Evidence and Guidlines for Management. 2. Auflage. Edinburgh/Toronto: Elsevier, 2009

16 Brown S, Gartland D, Perl S, McDonald E, MacArthur C. Conclution about urinary and faecal incontinence in the year after childbirth: a cohort study. BJOG 2105; 122: 954-962

17 NICE National Institute for Health and Care Excellence, UK. 2006. nice.org.uk. CG 37: Postnatal care: Routine postnatal care of women and their babies. [Online] 07 2006. [Zitat vom: 25. 03 2013.] http:// publications.nice.org.uk/postnatal-carecg37/guidance\#maternal-health.

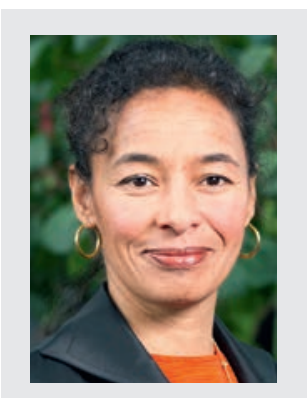

Osariere-Claudia Röthlisberger MSc Midwifery

E-Mail: osa.roe@bluewin.ch 